\title{
First-principles calculations on the structure of hydrogen aggregates in silicon and diamond
}

\author{
N Martsinovich ${ }^{1,3}$, M I Heggie ${ }^{1}$ and C P Ewels ${ }^{1,2}$ \\ ${ }^{1}$ CPES, University of Sussex, Falmer, Brighton BN1 9QJ, UK \\ 2 Département Matériaux et Systèmes Composites, ONERA, 29 avenue de la Division Leclerc, \\ 92322 Châtillon, France \\ E-mail: N.Martsinovich@sussex.ac.uk
}

Received 7 August 2003

Published 19 September 2003

Online at stacks.iop.org/JPhysCM/15/S2815

\begin{abstract}
We report the results of first-principles calculations on the early stages of hydrogen aggregation in silicon and diamond. We demonstrate that the hydrogenated glide dislocation dipole is the preferred structure for small numbers of $\mathrm{H}$ atoms in silicon and that it expands by dislocation glide, with hydrogen condensing in the shuffle plane between the dislocations. This structure is a good candidate for the initial stage in the development of hydrogeninduced platelets. We investigate the effect of shear and dilation on the energies of hydrogenated structures and compare the relative stabilities of these structures in silicon and diamond. We describe the method of determination of the Burgers vectors of dilation and shear for the dislocation dipoles by varying the lattice vectors of their supercells.
\end{abstract}

(Some figures in this article are in colour only in the electronic version)

\section{Introduction}

Hydrogen in silicon has been a subject of wide investigation because of its considerable effect on the properties of silicon and other semiconductors. Hydrogen binds to defects, such as impurity atoms, vacancies, dislocations and grain boundaries, passivates them and thus affects the electrical and optical properties of silicon [1,2].

Theoretical calculations confirm that hydrogen bonded to dangling bonds at defect sites has the lowest energy [3,4]. In defect-free silicon the $\mathrm{H}_{2}$ molecule at the tetrahedral interstitial site is the stable configuration [3-5]. The metastable configuration is the $\mathrm{H}_{2}^{*}$ defect, which consists of one $\mathrm{H}$ atom near the $\mathrm{Si}-\mathrm{Si}$ bond centre and the second $\mathrm{H}$ atom at the antibonding position [3].

3 Author to whom any correspondence should be addressed. 
Hydrogen introduced in high concentrations generates extended planar defects in silicon, lying along (111) and (100) crystallographic planes, that are called hydrogen-induced platelets (HIP) [6-13]. The platelets are located at depths corresponding to the maximum hydrogen concentration near the surface of the Si samples. Thus platelets are responsible for the formation of the hydrogen accumulation layer in silicon.

Platelets grow in size on annealing and, at high temperatures (provided a high enough concentration of hydrogen), develop into microcracks and lead to surface blistering of silicon samples [8-10]. This phenomenon is the basis of the technologically important SmartCut ${ }^{\mathrm{TM}}$ process of obtaining high-quality silicon-on-insulator (SOI) layers [14].

Spectroscopic investigations showed that platelets contain $\mathrm{Si}-\mathrm{H}$ bonds $[6,11-13]$ and, at $T>200{ }^{\circ} \mathrm{C}, \mathrm{H}_{2}$ molecules $[12,13]$. The temperature dependence of $\mathrm{Si}-\mathrm{H}$ and $\mathrm{H}_{2}$ vibrational modes suggests that several structures exist which transform into one another on annealing [11, 13]. The exact atomic structure of platelets is still a subject of investigation.

Theoretical calculations have been performed mainly on the structures of the (111) platelets in silicon [5, 15-18]. A number of possible platelet structures have been proposed, including hydrogen saturating broken $\mathrm{Si}-\mathrm{Si}$ bonds in the shuffle plane of the perfect silicon lattice, a similar structure containing $\mathrm{H}_{2}$ molecules, an aggregate of the $\mathrm{H}_{2}^{*}$ defects, a hydrogenated half-stacking fault structure [5, 15-17], and structures containing hydrogenated vacancies [18].

There is less detailed information on hydrogen on diamond compared with silicon. Theoretical calculations identified bond-centred hydrogen as the most stable position for one $\mathrm{H}$ atom in diamond [19] and the $\mathrm{H}_{2}^{*}$ complex for two $\mathrm{H}$ atoms [20]. Experimental studies demonstrate that hydrogen in diamond condenses mainly near grain boundaries [21]. IR absorption measurements suggest that, at low temperatures, a fraction of hydrogen in CVD diamond is present in an unbound form, and it was suggested that this forms $\mathrm{H}_{2}$ molecules [22]. A high concentration of hydrogen in the sub-surface area in diamond has been observed [23], suggesting that hydrogen is trapped there and forms some stable multi-H complexes [24]. One of the suggested [24] candidates for such complexes is the $\mathrm{H}_{2}^{*}$ complex. Another possibility is that hydrogen forms extended defects-platelets, similar to HIP observed in silicon. This view is supported by experimental observations of hydrogen-filled bubbles in diamond on annealing [25] and surface blistering of annealed proton-implanted diamond [10].

Theoretical investigations of hydrogen in silicon or diamond concentrate on small (oneor two-atom) hydrogen complexes [3-5, 19, 20] and infinite or large-scale hydrogen-related defects in silicon, such as HIP [15-18].

In this work we report the results of first-principles calculations on infinite HIP and medium-size hydrogen aggregates in silicon and diamond. The calculations were performed using $a b$ initio density functional code, AIMPRO, within the supercell approach [26]. Calculations of finite hydrogen aggregates in silicon were performed with $48 \mathrm{Si}$ atom cells. Some calculations were repeated using $96 \mathrm{Si}$ atom cells. Calculations on hydrogen in diamond involved $96 \mathrm{C}$ atom cells. Calculations on infinite HIP were performed with $12 \mathrm{Si}$ atom cells. Some calculations were repeated using 24 atom cells; their results agreed with those performed in small cells. The Brillouin zone was modelled using the Monkhorst-Pack sampling scheme with four or eight $k$-points.

We investigate the stabilities of various possible structures of infinite HIP for a range of lattice dilations. Then we discuss the initial stages of condensation of hydrogen in silicon, leading to the formation of large $\mathrm{H}$ aggregates and the development of platelets. We compare the energies of several possible hydrogen complexes with different numbers of $\mathrm{H}$ atoms. We find local shear and dilation associated with these complexes and discuss their effect on the energies. Calculations of hydrogen aggregation in diamond follow similar calculations in silicon. 


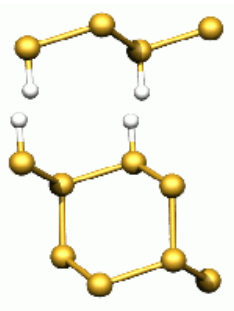

$[2 \mathrm{Si}-\mathrm{H}]_{\mathrm{n}}$

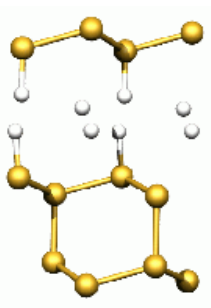

$\left[2 \mathrm{Si}-\mathrm{H}+\mathrm{H}_{2}\right]_{\mathrm{n}}$

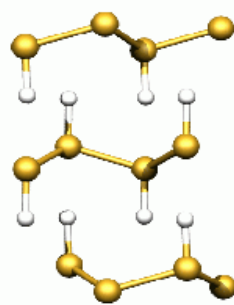

$\left[\mathrm{H}_{2}^{*} \mathrm{I}_{\mathrm{n}}^{\mathrm{D}}\right.$

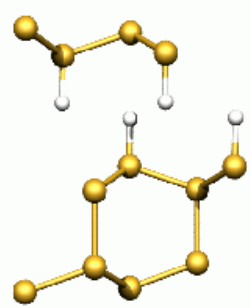

half-stacking fault

Figure 1. Possible structures of HIP in silicon

\section{Results}

\subsection{Infinite hydrogen-induced platelets in silicon}

Calculations of the stabilities of various structures of platelets have been reported in the literature [5, 15-18]. Among the structures that were considered were an array of bondcentred hydrogen atoms $[5,15]$, aggregates of the $\mathrm{H}_{2}^{*}$ defects in a single or double layer [16, 17], hydrogen saturating broken $\mathrm{Si}-\mathrm{Si}$ bonds in the shuffle plane and the same structure with $\mathrm{H}_{2}$ molecules between the (111) planes [15, 17], and the half-stacking fault structure described above [16]. Zhang and Jackson [16] found the half-stacking fault structure to be the lowestenergy structure but discarded it due to the high barrier of formation and the need for preexisting defects such as dislocations for its formation. Thus they considered a double-layer $\mathrm{H}_{2}^{*}$ aggregate as the most stable structure.

The effect of lattice dilation in the direction normal to the platelet on the energies of these structures was considered [16, 17]. The energies of all the structures were found to decrease with dilation, which is consistent with the experimental result that the lattice around the platelet is dilated by $\sim 3 \AA$ [6]. According to Kim and Chang [17], the lowest-energy structure changed from $\left[\mathrm{H}_{2}^{*}\right]_{n}^{D}$ at small dilations to $\left[\mathrm{Si}-\mathrm{H}+\mathrm{H}_{2}\right]_{n}$ at large dilations.

We calculate the energies of a number of structures - the double layer of $\mathrm{H}_{2}^{*} \mathrm{~s}\left(\left[\mathrm{H}_{2}^{*}\right]_{n}^{D}\right.$ in Kim and Chang's notation), the half-stacking fault, $\mathrm{Si}-\mathrm{H}$ bonds in the shuffle plane with and without $\mathrm{H}_{2}$ molecules (structures $[\mathrm{Si}-\mathrm{H}]_{n}$ and $\left[\mathrm{Si}-\mathrm{H}+\mathrm{H}_{2}\right]_{n}$, respectively, see figure 1) - as a function of lattice dilation. In these calculations we use $12 \mathrm{Si}$ atom supercells. These cells repeated, in 3D form, layers of infinite platelets.

The results are shown in figure 2 . The energies of all the structures decrease with dilation, in agreement with the previous theoretical works [16, 17]. We find the half-stacking fault structure omitted in earlier investigations to be the lowest-energy one at small dilations below $1.5 \AA$, lower than the $\left[\mathrm{H}_{2}\right]_{n}^{D}$. At larger dilations the structure with $\mathrm{Si}-\mathrm{H}$ bonds in the shuffle 


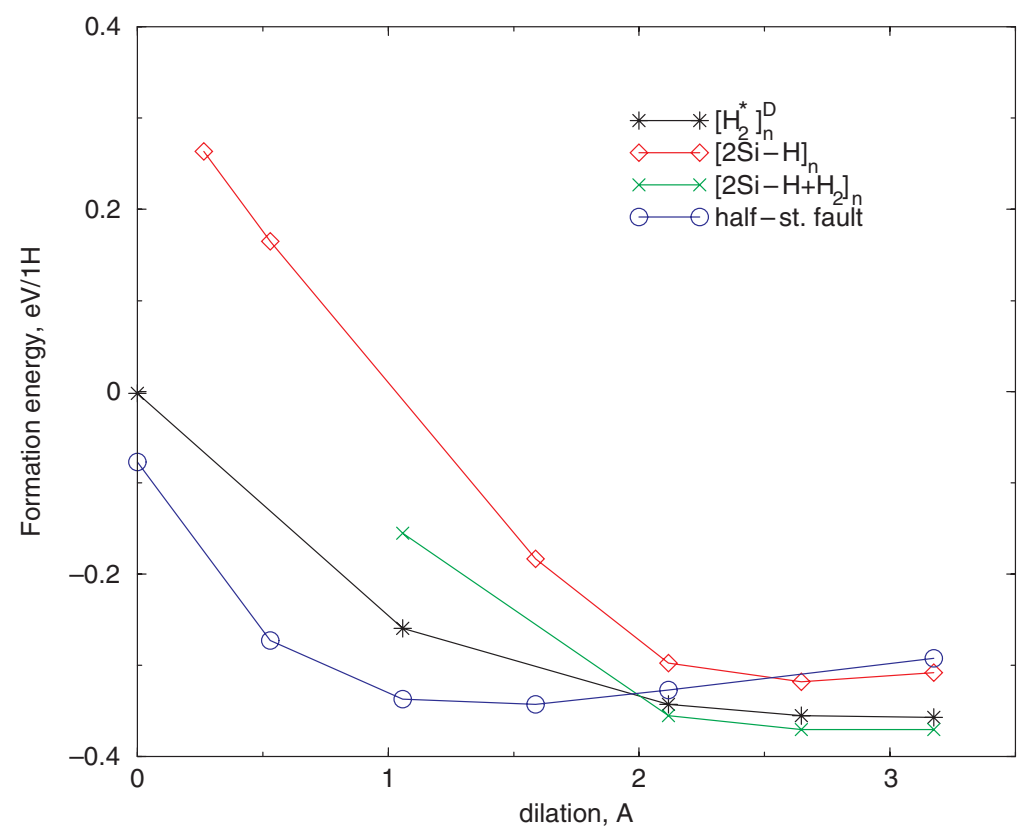

Figure 2. The formation energies of various possible platelet structures with respect to an $\mathrm{H}_{2}$ molecule at the $\mathrm{T}_{\mathrm{d}}$ site.

plane and $\mathrm{H}_{2}$ molecules becomes most stable. We consider the half-stacking fault structure to be valid for small-size platelets where only relatively small dilations are possible, as will be obvious from our calculations for finite-size platelets reported in the next section. We believe that, as platelets grow larger and larger dilations are possible, their structure changes to the structure with $\mathrm{Si}-\mathrm{H}$ bonds and $\mathrm{H}_{2}$ molecules in the shuffle plane. This structure is the global energy minimum and is consistent with the experimental determination of precise atomic positions using high-resolution transmission electron microscopy [6] and with spectroscopic determination of the dielectric constant of the high-temperature platelet structure [13].

\subsection{Finite-size hydrogen aggregates in silicon}

As the simplest system we consider the aggregate of $\mathrm{H}$ atoms that is infinite in one dimension (along the $z$-axis-see figure 3), with four $\mathrm{H}$ atoms per repeat distance.

The choice of structures was influenced by our earlier result for diamond [27], where a hydrogenated faulted $90^{\circ}$ glide dislocation loop was found to be the lowest-energy configuration for four $\mathrm{H}$ atoms in diamond compared with two hydrogenated neighbouring $\langle 111\rangle$ and $\langle 110\rangle \mathrm{C}-\mathrm{C}$ bonds. The hydrogenated dislocation loop was formed spontaneously from a pair of $\mathrm{H}_{2}^{*}$ defects, the lowest-energy structure for two $\mathrm{H}$ atoms in diamond.

Our similar calculations on hydrogen in silicon show that, in the case of four $\mathrm{H}$ atoms in silicon, the hydrogenated glide $90^{\circ}$ dislocation loop is the lowest-energy structure as well$1.9 \mathrm{eV}$ lower than two hydrogenated neighbouring $\langle 111\rangle \mathrm{Si}-\mathrm{Si}$ bonds and nearly degenerate with a pair of $\mathrm{H}_{2}$ molecules in the perfect silicon lattice. Hydrogenation significantly reduces the formation energy of a dislocation loop in silicon, which equals $2.7 \mathrm{eV}$ for a non-hydrogenated dislocation loop according to our supercell calculations.

Therefore one of the structures for extended hydrogen aggregates in silicon that was studied was the hydrogenated glide dislocation dipole (figure 3(a)). The other structure investigated 


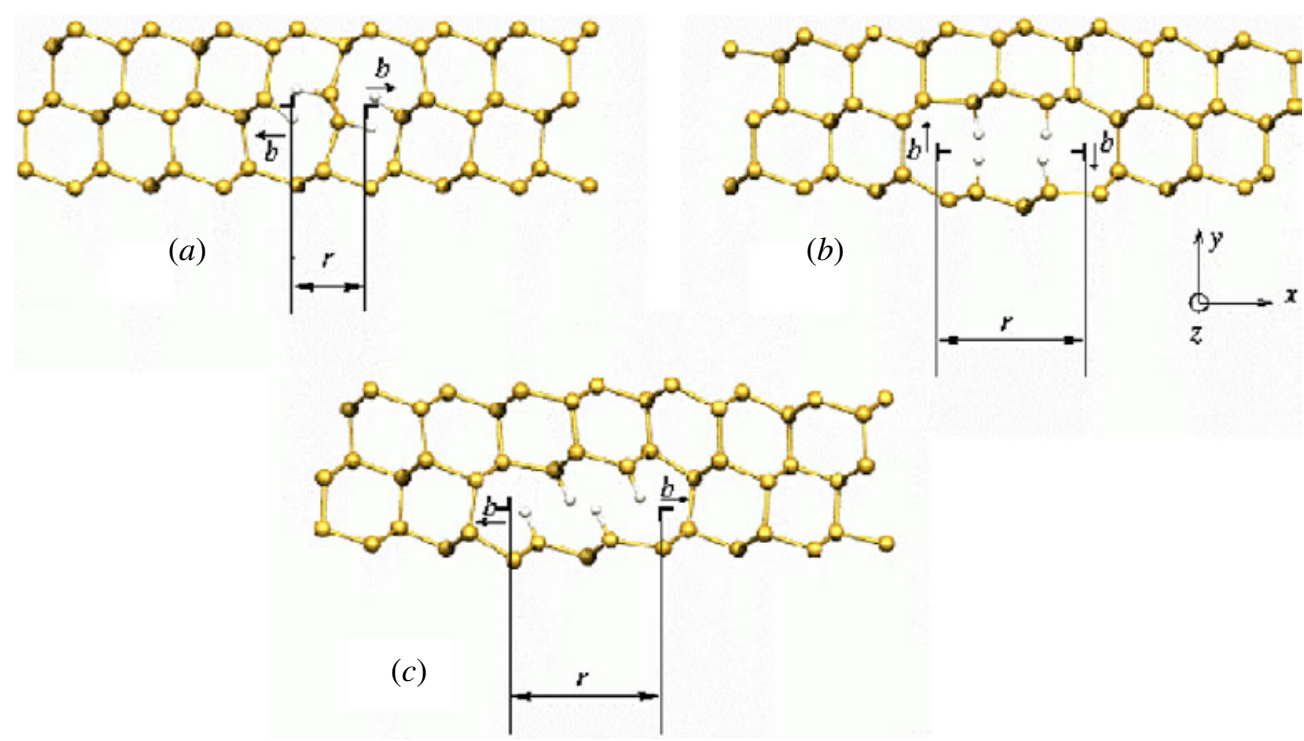

Figure 3. Examples of 48 silicon atom cells with a small aggregate of $\mathrm{H}$ atoms: (a) hydrogenated glide dislocation dipole; (b) Si-H bonds along the $\langle 111\rangle$ direction (unstable); and (c) hydrogenated shuffle dislocation dipole. Burgers vectors $b$ and separations of dislocation cores $r$ are shown.

Table 1. The energies of hydrogenated dislocation dipoles obtained by first-principles calculations and using elasticity theory equations. First-principles energies are given for $48 \mathrm{Si}$ atom cells.

\begin{tabular}{llll}
\hline & \multicolumn{2}{c}{ Ab initio } & \\
\cline { 2 - 3 } $\begin{array}{l}\text { Energy differences } \\
(\mathrm{eV} / \text { repeat distance })\end{array}$ & $\begin{array}{l}\text { Lattice vectors as } \\
\text { in perfect silicon }\end{array}$ & $\begin{array}{l}\text { Shear and dilation } \\
\text { applied to the cells }\end{array}$ & Elasticity theory \\
\hline$E$ (shuffle) $-E$ (glide) & 1.59 & 0.98 & 1.42 \\
$E($ dil $)-E($ glide $)$ & 2.80 & 1.53 & 1.58 \\
\hline
\end{tabular}

was the configuration with $\mathrm{Si}-\mathrm{H}$ bonds facing each other along the $\langle 111\rangle$ direction (figure $3(b)$ ). This can be considered as a dipole of edge dislocations with Burgers vectors in the $\langle 111\rangle$ direction and 'extra material' effectively appearing in the platelet region (a dilational dipole). This configuration was found to be unstable in small-size platelets and relaxed by shear along the $\langle 112 \underline{2}\rangle$ direction, giving a $90^{\circ}$ dislocation dipole in the shuffle plane (figure $3(c)$ ).

The hydrogenated glide dislocation dipole had the lowest energy among these three structures-1.59 (1.72) eV lower in energy than the shuffle dislocation dipole and 2.80 (2.73) eV lower than the dilational dipole in 96 (48)-atom cells (see table 1-energies are given per repeat distance along the dislocation line, $3.84 \AA$ ). Note that the energy of the dilational dipole is the approximate energy of the intermediate structure before the shear relaxation. For the larger cells, energy differences between the structures reduced. We believe that this is due to the increase in dilation allowed for the shuffle platelet.

A well-known problem arising in supercell calculations with cells containing dislocation dipoles is that lattice mismatch due to these dislocation dipoles appears at the cell boundaries [28]. Lehto and Oberg [29] suggested a way to avoid this mismatch by adjusting the lattice vectors of the supercell in accordance with the lattice vectors and respective positions of dislocations in a dipole, so that, for example, when two oppositely signed edge dislocations 
in the same glide plane are present in the perfect material, the cells are displaced with respect to each other by $b \times(w / L)$ in the direction of glide (where $b$ is the value of the Burgers vector of a dislocation, $w$ is the inter-dislocation distance, and $L$ is the length of the cell).

This approach was applied in our calculations to the cells containing hydrogenated dislocation dipoles. A range of possible values of $b$ was tried. The dependence of the energies of the hydrogenated shuffle dipole on the Burgers vector of shear in the $x$-direction exhibits a minimum. Fitting this dependence to a quadratic function gave the value of $1.8 \AA$ for the Burgers vector of shear for the shuffle dislocations in silicon. This value is close to $2.22 \AA$, the Burgers vector of a $90^{\circ}$ glide partial dislocation in silicon.

Dilation of the cell containing a hydrogenated shuffle dipole was found to decrease its energy as well, with an energy minimum corresponding to dilation by $1.1 \AA \times(w / L)$. This indicates that this dipole is not purely shear but has a partly dilational character. Or, in other words, the Burgers vector of the shuffle dislocation has a shear component $b_{\text {sh }}$ and a dilational component $b_{\text {dil }}$.

Since both dilation and shear reduce the energy of this structure, the global energy minimum should correspond to a combination of dilation and shear. Indeed, comparing the energies of this structure for several values of shear and dilational Burgers vectors shows that the minimum energy corresponds to displacement of the cells along the $x$-direction by $1.8 \AA \times(w / L)$ and dilation by $1.1 \AA \times(w / L)$. The total Burgers vector of a shuffle dislocation is a combination of $b_{\text {sh }}$ and $b_{\text {dil }}$ and equals $2.1 \AA$.

Thus, the described approach of calculating the energies of cells containing dislocation dipoles for a range of cell displacements and dilations allows us to find the optimum Burgers vectors of dislocation dipoles, which give the minimum energy for the structures under consideration.

The cell that contains a dilational dipole was dilated in a similar way by $b_{\text {dil }} \times(w / L)$, where $b_{\text {dil }}$ varied. The energies of the structures at intermediate points of relaxation, before the appearance of shear, were extracted. Their dependence on dilation was fitted to a quadratic function. The position of the energy minimum gave the approximate value of the optimum dilational Burgers vector, $1.8 \AA$.

Displacement was applied to cells with hydrogenated glide dipoles as well. Unlike the case of shuffle dipoles, a displacement of cells by $b \times(w / L)$ was found to slightly increase the energy of this structure $(0.09 \mathrm{eV}$ for dilation by $2.22 \AA \times(w / L))$. However, when similar treatment was applied to the non-hydrogenated glide dipole, it behaved in a way similar to the hydrogenated shuffle dipole and exhibited a dependence with a minimum at $b=2.2 \AA$, which is in very good agreement with the accepted value of $2.22 \AA$ in the literature. Therefore the energy of the hydrogenated glide dipole in the cell displaced by $2.22 \AA \times(w / L)$ was further compared with the energies of the structures discussed above with hydrogen in the shuffle plane.

Table 1 summarizes the energies of the structures discussed as well as the effect of cell displacement and shear. It is seen clearly from the table that the energies of the shear and dilational hydrogenated dipoles are significantly reduced when the lattice vectors are optimized. However, the order of stabilities remains the same: the hydrogenated glide dipole is the lowestenergy structure, $1 \mathrm{eV}$ lower than the shuffle dipole and $1.5 \mathrm{eV}$ lower than the dilational dipole.

The energy differences between different finite platelet structures appear to be controlled by the elastic interactions of the dislocations bounding the platelets. The elastic energy of a dipole of $90^{\circ}$ dislocations is given by the following equation [30]:

$$
E=\frac{\mu b^{2}}{2 \pi(1-v)}\left[\ln \frac{w}{r_{0}}-\frac{1}{2} \cos 2 \theta\right]
$$


where $\mu$ is the shear modulus, $v$ is Poisson's ratio, $b$ is the magnitude of the Burgers vector, $w$ is the distance between the dislocations, $r_{0}$ is the dislocation core radius, and $\theta$ is the angle between the glide plane of one dislocation and the direction to the other dislocation.

Equation (1) shows that the energy of a dislocation dipole depends on the inter-dislocation distance and on the character (shear or dilational) of the dipole: the more narrowly separated dipoles (like the glide dipole in figure 3(a)) should be lower in energy, and dipoles with larger dilational components should be higher in energy than shear dipoles due to the effect of the angular term $\cos 2 \theta$.

Using Burgers vectors found by fitting for the shuffle and dilational dipoles as well as the value of $2.22 \mathrm{~A}$ in the literature for the Burgers vector of the glide dipole, we find the shear dipole in figure $3(c)$ to be $1.42 \mathrm{eV}$ higher in energy than the glide dipole (figure $3(a)$ ) and the dilational dipole (figure $3(b)$ ) to be $1.58 \mathrm{eV}$ higher than the glide dipole, if we treat dislocation dipoles as being isolated and neglect interactions with image dipoles which appear in the supercell method. This approach is valid for narrowly separated dipoles in large cells.

In table 1 the energies of dislocation dipoles calculated using elasticity theory are compared with the results of first-principles calculations. The table shows that the energy differences between the dipoles predicted by elasticity theory are in reasonable agreement with the energies obtained from $a b$ initio calculations. This shows that elastic interactions of dislocations bounding the platelets are the most important single factor controlling the stabilities of small platelets.

The other factor that should be taken into consideration in order to obtain agreement with $a b$ initio results is the different energies of hydrogen bonding to silicon in these structures.

On addition of more hydrogen, the lowest-energy structure- - the hydrogenated dislocation dipole in the glide plane-can expand by glide. It has been shown experimentally that dislocation glide is facilitated by hydrogenation of dislocation cores with the activation energy of dislocation motion in silicon reducing from 2.2 to $1.2 \mathrm{eV}$ (i.e. the hydrogen-enhanced dislocation glide, or HEDG effect) [31].

The extra hydrogen fills the space between the dislocations, forming $\mathrm{Si}-\mathrm{H}$ bonds in the shuffle plane. This structure is similar to the half-stacking fault structure proposed by Zhang and Jackson [16]. In this structure the elastic field of the glide dislocation dipole is counteracted by shear created by hydrogen accumulated in the shuffle plane in the stacking fault.

As an example, the structure containing $12 \mathrm{H}$ atoms per repeat distance (figure $4(a)$ ) was calculated and its energy was compared with other possible configurations with the same numbers of $\mathrm{H}$ atoms per cell: the aggregate of $\mathrm{H}_{2}^{*}$ defects in the (111) plane (figure $4(b)$ ); the glide dislocation dipole with extra hydrogen forming $\mathrm{Si}-\mathrm{H}$ bonds in the glide plane (figure $4(c)$ ); and the hydrogenated shuffle dipole (figure $4(d)$ ). Among them, the 'half-stacking fault' structure had the lowest energy $-0.34 \mathrm{eV}$ lower than the aggregate of $\mathrm{H}_{2}^{*} \mathrm{~s}, 0.51 \mathrm{eV}$ more stable than the glide dipole, and $4.04 \mathrm{eV}$ more stable than the shuffle dipole.

In order to obtain more consistent energies, we tested the effect of shear and dilation on the two lowest-energy structures. Dilation was found to reduce the energies of both structures. The optimum dilational Burgers vector was $1.2 \AA$ for the half-stacking fault structure and $1.5 \AA$ for the $\mathrm{H}_{2}^{*}$ aggregate. The larger dilation needed for the $\mathrm{H}_{2}^{*}$ structure can be understood by comparing figures $4(a)$ and $(b)$ : the $\mathrm{H}_{2}^{*}$ complex has four layers of $\mathrm{Si}-\mathrm{H}$ bonds compared with two layers in the half-stacking fault structure, therefore it needs larger dilations to accommodate hydrogen more efficiently. The half-stacking fault structure remained the more stable structure when dilation was applied $-0.20 \mathrm{eV}$ lower than the $\mathrm{H}_{2}^{*}$ aggregate.

We regard the half-stacking fault structure as the structure arising from the dislocation dipole in the glide plane and, in addition, containing the second shear dipole in the shuffle plane. We expect the shear due to these two dipoles to approximately cancel each other, resulting in 


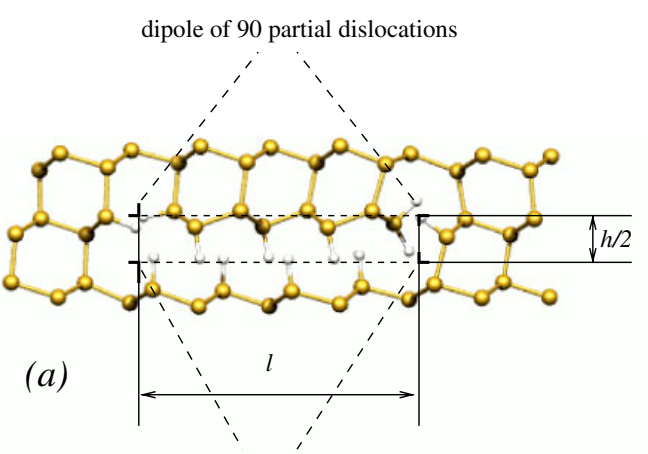

$\mathrm{H}$ in the shuffle plane creates shear->

formation of the second dislocation dipole

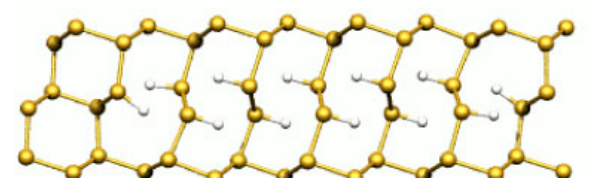

(c)

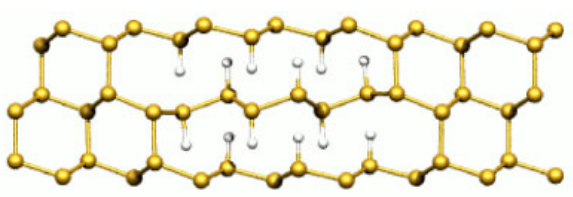

(b)

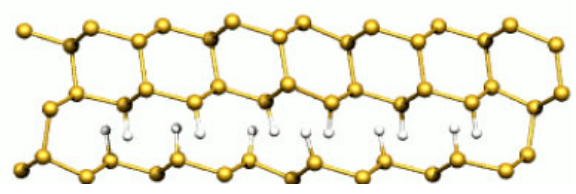

(d)

Figure 4. Examples of 48 silicon atom cells with $12 \mathrm{H}$ atoms in a cell: ( $a$ ) half-stacking fault structure; $(b)$ aggregate of $\mathrm{H}_{2}^{*}$ defects; $(c)$ hydrogenated glide dislocation dipole; and (d) hydrogenated shuffle dislocation dipole.

no total shear associated with this structure. Indeed, our $a b$ initio calculations show that shear applied to the cells does not reduce the energy of this structure. More exactly, the minimum of the dependence of $E$ on shear corresponds not to $b_{\text {sh }}=0$ but to $0.15 \AA$. This supports our finding that the Burgers vector of shear of the hydrogenated shuffle dipole is slightly smaller than the Burgers vector of the glide $90^{\circ}$ dislocation dipole.

Thus, we find the half-stacking fault structure to be the most stable structure for mediumsize hydrogen aggregates in silicon and consider it as the preferred structure for HIP at the initial stages of their formation, assuming thermal equilibrium at each step.

\subsection{Hydrogen aggregates in diamond}

The medium-size aggregates of hydrogen in diamond were also studied. We considered the same structures as those discussed above for silicon.

$A b$ initio calculations of energies of the structures with four $\mathrm{H}$ per repeat distance show that the dilational dipole is unstable, like in silicon, and is approximately $2.4 \mathrm{eV}$ higher in energy than the hydrogenated glide dipole, while the hydrogenated glide and shuffle dipoles are degenerate in $96 \mathrm{C}$ atom cells. In $48 \mathrm{C}$ atom cells the shuffle dipole is relatively higher in energy and is $0.58 \mathrm{eV}$ less stable than the glide dipole. This difference in energies obtained using cells of different sizes is qualitatively similar to the results for silicon mentioned in the previous section, but in the case of diamond the effect of the cell size is much more pronounced. Thus dilation, which is more feasible in large cells than in small cells, is very important for $\mathrm{H}$ aggregates in diamond. 
Adjustment of the lattice vectors showed that the character of the shuffle dipole is more dilational than shear: the dilational component of the Burgers vector is $1.4 \AA$ and the shear component is $0.5 \AA$. Adjusting the lattice vectors made the shuffle dipole $1.84 \mathrm{eV}$ more stable than the glide dipole, unlike the situation for silicon. This may indicate that the initial stages of hydrogen aggregation in diamond proceed in a different way to that in silicon.

Larger hydrogen aggregates in diamond, namely the half-stacking fault structure, the aggregate of $\mathrm{H}_{2}^{*} \mathrm{~s}$ and the hydrogenated shuffle dipole, were also considered. The half-stacking fault structure was decisively more stable than the other structures- $4.22 \mathrm{eV}$ lower than the $\mathrm{H}_{2}^{*}$ aggregate and $6.12 \mathrm{eV}$ lower than the shuffle dipole.

Thus, for medium-size hydrogen aggregates in diamond the half-stacking fault structure involving the dislocation dipole in the glide plane and the accumulation of hydrogen in the shuffle plane associated with shear is the preferred structure, similar to the case for hydrogen in silicon. This structure is a likely candidate for the precursor in the further aggregation of hydrogen in diamond.

\section{Conclusions}

Our calculations show that small numbers of hydrogen atoms in silicon have a tendency to aggregate, forming hydrogenated glide dislocation dipoles. We demonstrate that small hydrogen aggregates with different structures can be described in terms of dislocations, and that the main factor controlling their relative stabilities is the dislocation interaction energies. Structures involving shear are energetically favoured compared to structures involving dilation.

We show that the adjustment of lattice vectors in supercell calculations according to the type of dislocations contained in the cells is an important factor in determining the stabilities of different structures. We investigated the effect of changing the lattice vectors, i.e. dilation and shear, on the energies of hydrogenated structures, and showed that lattice dilation has a stronger effect on the stabilities of hydrogen aggregates in diamond, favouring the structures that are capable of dilation and reversing the relative stabilities of the glide and shuffle dipoles observed for silicon.

On the basis of our calculations, we suggest that incipient platelets growing under quasiequilibrium conditions would grow by the formation and movement of glide dislocations that bound a half-stacking fault structure. As they expand, dilation becomes favoured and the stacking fault is removed by dislocation glide (enhanced by the presence of hydrogen), yielding larger structures of $\left[\mathrm{Si}-\mathrm{H}+m \mathrm{H}_{2}\right]_{n}$ type, which is consistent with HRTEM measurements of atomic positions [6] and with the spectroscopically determined dielectric constant of the hightemperature platelet structure [13].

\section{References}

[1] Pearton S J, Corbett J W and Shi T S 1987 Appl. Phys. A 43153

[2] Pankove J I and Johnson N M (ed) 1991 Hydrogen in Semiconductors (Semiconductors and Semimetals vol 34) (Boston, MA: Academic)

[3] Chang K J and Chadi D J 1989 Phys. Rev. B 4011644

[4] Van de Walle C G 1994 Phys. Rev. B 494579

[5] Van de Walle C G, Denteneer P J H, Bar-Yam Y and Pantelides S T 1989 Phys. Rev. B 3910791

[6] Johnson N M, Ponce F, Street R A and Nemanich R J 1987 Phys. Rev. B 354166

[7] Muto S, Takeda S and Hirata S 1995 Phil. Mag. A 721057

[8] Grisolia J, Cristiano F, Ben Assayag G and Claverie A 2001 Nucl. Instrum. Methods B 178160

[9] Weldon M K, Marsico V E, Chabal Y J, Agarwal A, Eaglesham D J, Sapjeta J, Brown W L, Jacobson D C, Caudano Y, Christman S B and Chaban E E 1997 J. Vac. Sci. Technol. B 151065 
[10] Tong Q Y, Gutjahr K, Hopfe S, Gösele U and Lee T-H 1997 Appl. Phys. Lett. 701390

[11] Heyman J N, Ager J W III, Haller E E, Johnson N M, Walker J and Doland C M 1992 Phys. Rev. B 4513363

[12] Leitch A W R, Alex V and Weber J 1998 Solid State Commun. 105215

[13] Lavrov E V and Weber J 2001 Physica B 308151

[14] Bruel M 1995 Nucl. Instrum. Methods B 108313

[15] Deak P, Ortiz C R, Snyder L C and Corbett J W 1991 Physica B 170223

[16] Zhang S B and Jackson W B 1991 Phys. Rev. B 4312142

[17] Kim Y S and Chang K J 2001 Phys. Rev. Lett. 861773

[18] Reboredo F A, Ferconi M and Pantelides S T 1999 Phys. Rev. Lett. 824870

[19] Estle T L, Estreicher S and Marynick D S 1987 Phys. Rev. Lett. 581547

[20] Briddon P R, Jones R and Lister G M S 1988 J. Phys. C: Solid State Phys. 21 L1027

[21] Zhou X, Watkins G D, Rutledge K M M, Messmer R P and Chawla S 1996 Phys. Rev. B 547881

[22] Khomich A V, Ralchenko V G, Vlasov A V, Khmelnitsky R A, Vlasov I I and Konov V I 2001 Diamond Relat. Mater: 10546

[23] Ballutaud D, Jomard F, Theys B, Mer C, Tromson D and Bergonzo P 2001 Diamond Relat. Mater 10405

[24] Connell S H, Sellschop J P F, Butler J E, Maclear R D, Doyle B P and Machi I Z 1998 Diamond Relat. Mater. 7 1714

[25] Gippius A A, Khmelnitsky R A, Dravin V A and Khomich A V 2001 Physica B 308-310 573

[26] Coutinho J, Jones R, Briddon P R and Öberg S 2000 Phys. Rev. B 6210824

[27] Heggie M I, Ewels C P, Martsinovich N, Scarle S, Jones R, Goss J P, Hourahine B and Briddon P R 2002 J. Phys.: Condens. Matter 1412689

[28] Bigger J R K, McInnes D A, Sutton A P, Payne M C, Stich I, King-Smith R D, Bird D M and Clarke L J 1992 Phys. Rev. Lett. 692224

[29] Lehto N and Oberg S 1998 Phys. Rev. Lett. 805568

[30] Cottrell A H 1965 Dislocations and Plastic Flow in Crystals (Oxford: Clarendon)

[31] Yamashita Y, Kyobe F, Kamiura Y and Maeda K 1999 Phys. Status Solidi a 17127 